03

\title{
Влияние начальных условий на скорость фронта ламинарного пламени в газовых смесях
}

\author{
(С) В.В. Володин, В.В. Голуб, А.Е. Ельянов \\ Объединенный институт высоких температур РАН, \\ 125412 Москва, Россия \\ e-mail: golub@ihed.ras.ru
}

Поступило в Редакцию 29 июня 2020 г.

В окончательной редакции 6 августа 2020 г.

Принято к публикации 11 августа 2020 г.

Рассмотрен разброс скоростей фронта ламинарного пламени, вызванный как погрешностью в составлении горючей смеси, так и искусственными начальными возмущениями. Показано, как конфигурация начальных возмущений изначально плоского фронта ламинарного пламени в газовой смеси постоянного состава влияет на разброс скоростей расширяющихся сферических пламен. Для литературных данных по разбросу скорости распространения пламени выполнен анализ влияния погрешности в составлении горючей смеси на параметры, определяющие скорость фронта пламени. Эти параметры были пересчитаны для возможного разброса состава смеси, полученного на основе данных о точности оборудования, использованного в экспериментах.

Ключевые слова: ламинарное пламя, газовая смесь, искровый разряд, фронт пламени.

DOI: 10.21883/JTF.2021.02.50358.215-20

\section{Введение}

Распространение фронта экзотермической реакции в однородных неподвижных газовых смесях является базовой задачей для тестирования различных моделей в областях энергетики и двигателестроения [1,2]. Наибольший интерес представляют неустойчивые режимы распространения фронта реакции, сопровождающиеся осцилляциями параметров и ускорением процесса выделения энергии.

Для достоверных расчетов ускорения фронта реакции необходимо знание законов его распространения. В целом процессу воспламенения уделяется немало внимания, сложность описания самого процесса, а также разнообразие способов воспламенения [3] активно обсуждается в научной периодике. Ранее авторами было показано, что экспериментальные данные по динамике фронта пламени для одной смеси при неизменных условиях могут меняться от эксперимента к эксперименту $[4,5]$. Проблема различия в экспериментальных данных по ускорению пламени в каналах, скорости сферического распространения пламени и скорости ламинарного горения в газовых смесях, полученных разными исследователями, не раз поднималась в литературе $[6,7,8]$. Несмотря на все усилия, направленные на повышение точности создания объекта исследований и измерения его параметров, существенного снижения разброса не наблюдалось. Важность учета стохастической природы ускорения пламен указывалась в научных публикациях при рассмотрении турбулентного горения $[9,10]$. В последнее время все больше исследователей склоняются к вероятностному описанию не только турбулентных режимов горения, но и процессов воспламенения [11] и распространения неустойчивых ламинарных пламен [1]. В работе [1] авторы провели серию экспериментов по воспламенению и исследованию начального этапа распространения пламени в смесях водорода, этилена и пропана с воздухом и получили разброс скоростей. Причины полученного разброса будут проанализированы далее.

Одним из способов численного и аналитического описания скорости распространения и формы фронта экзотермической реакции является интегро-дифференциальное уравнение Сивашинского [12]. Уравнение описывает эволюцию поверхности, соответствующей фронту химической реакции, толщина которого считается бесконечно малой в сравнении с характерными размерами неоднородностей. Изначально сформулированное для слабонелинейного плоского фронта пламени уравнение было адаптировано для описания расширяющегося пламени [13] и сильно нелинейного пламени, неустойчивого по термодиффузионному механизму [14].

Построение динамики распространения фронта пламени путем численного решения уравнения Сивашинского впервые было проведено Д. Михельсоном и Г. Сивашинским в плоской двумерной постановке [15]. В дальнейшем уравнение Сивашинского решалось в двумерной постановке для расширяющегося пламени численно [16,17] и аналитически [18]. В работе [19] уравнение было дополнено усложненной моделью химической кинетики процесса. Работа [20] посвящена анализу зависимости коэффициента складчатости пламени от времени и концентрации водорода в водородно-воздушной смеси с помощью численного интегрирования уравнения Сивашинского в плоской двумерной постановке с длиной фронта пламени около $2 \mathrm{~m}$. Коэффициентом складча- 
тости („folding factor“ или „wrinkling factor“) $A / A_{0}$ называется отношение площади поверхности складчатого неустойчивого фронта пламени к площади, аналогичной сглаженной поверхности. Начальное распределение возмущений в работе [20] моделировалось „белым шумом“ со средней амплитудой $10^{-7} \mathrm{~m}$. Результаты демонстрируют для всех смесей характерный тип зависимости коэффициента складчатости от времени: в начальный момент и на протяжении некоторого периода индукции коэффициент складчатости мало отличается от единицы; затем за короткое время увеличивается до значений $1.3-1.4$; после этого начинаются колебания в пределах $10 \%$ величины с общей тенденцией к росту. Так же в работе [20] показано, что уменьшение амплитуды начальных возмущений приводит к росту периода индукции. Иные конфигурации начальных возмущений в этой работе не рассматривались.

В настоящей работе рассматривается представленный в работе [1] разброс скоростей фронта ламинарного устойчивого пламени, вызванный погрешностью в составлении горючей смеси, и разброс скоростей фронта ламинарного неустойчивого пламени, вызванный различием в конфигурации начальных возмущений.

\section{1. Анализ причин разброса скоростей в работе [1]}

В работе [1] был обнаружен разброс скоростей расширяющихся сферических пламен с радиусом до $8 \mathrm{~mm}$. Составы смесей $(23.3 \%$ водорода и $76.7 \%$ воздуха; $8 \%$ этилена и $92 \%$ воздуха; $5.2 \%$ пропана и $94.8 \%$ воздуха) были выбраны из условий наибольшей вероятности зажигания разрядом с энергией, близкой к минимальной [21]. Расстояние между электродами для каждой смеси выбиралось из соображений создания энергии, кратной минимальной энергии инициирования, и составляло $0.5 \mathrm{~mm}$ для водородно-воздушной, $1.2 \mathrm{~mm}$ для этилено-воздушной и $1.7 \mathrm{~mm}$ для пропано-воздушной смесей. Для каждой смеси и энергии инициирования было проведено по 5 повторений, когда происходило зажигание смеси. Перед каждым повторением горючая смесь готовилась с помощью регулятора-расходомера Bronkhorst EL-FLOW и контролировалась с помощью анализатора кислорода Servomex MiniHD 5200.

Точность регуляторов-расходомеров Bronkhorst EL-FLOW составляет $0.5 \%$ от величины показания и $0.1 \%$ от верхнего предела измерения [22]. Минимальная погрешность обеспечивается, когда измеряемая величина близка к верхнему пределу измерения, таким образом, содержание горючего в приготовленных смесях составляет: водорода $23.3 \pm 0.22 \%$, этилена $8 \pm 0.09 \%$, пропана $5.2 \pm 0.06 \%$. Анализатор кислорода Servomex MiniHD 5200 в исполнении с повышенной точностью имеет погрешность $0.05 \%$ [23]. При измерении концентрации кислорода в горючей смеси с заявленной погрешностью, погрешность измерения содержания горючего составит $0.24 \%$ для всех смесей. Соответственно проверка состава смеси анализатором Servomex MiniHD 5200 не снижает общей погрешности состава смеси.

Погрешность в составлении горючей смеси приводит к возможному различию таких параметров, определяющих скорость фронта пламени, как скорость ламинарного горения $S_{L}$, коэффициент расширения продуктов сгорания $\Theta$, температура продуктов сгорания, число Льюиса $L e$, число Зельдовича $Z e$ и длина Маркштейна $L_{M}$. Эти величины были пересчитаны для возможного разброса состава смеси. Значения скорости ламинарного горения, числа Льюиса и эффективного значения энергии активации для вычисления числа Зельдовича водородно-воздушных и пропано-воздушных смесей были взяты из работы [24]. Значение энергии активации для вычисления числа Зельдовича этилено-воздушных смесей было взято из [25]. Значения адиабатической температуры продуктов сгорания рассчитывались с использованием механизмов GRI-Mech 3.0 [26]. Значения скорости ламинарного горения этилено-воздушных смесей было взято из [27]. Числа Льюиса этиленовоздушных смесей рассчитывались по методике [28]. Значения длины Маркштейна водородно-воздушных смесей взяты из работы [29], этилено-воздушных — из [30], пропано-воздушных - из [31]. Соответствующие величины представлены в табл. 1.

На начальном этапе сферического распространения пламя стабильно. Начальные возмущения, вызванные неоднородностью поджигающего разряда, естественными флуктуациями состава, температуры и плотности горючей смеси, подавляются механизмами вязкости и теплопроводности [32]. Неустойчивость фронта пламени в смесях водорода и углеводородов с воздухом и его склонность к самоускорению начинается при достижении определенного радиуса пламени, характеризующегося первым критическим числом Пекле [33]:

$$
P e_{c}=31 M a_{b}+952, \quad-25<M a_{b}<30,
$$

где $M a_{b}$ - число Маркштейна.

Формула (1) получена из обработки экспериментальных данных и не учитывает стохастичности образования ячеистой структуры на фронте пламени, поэтому может использоваться только для оценки по порядку величины. В табл. 2 приведены значения критического радиуса пламени для рассматриваемых смесей.

В работе приведены зависимости радиуса фронта пламени от времени, при этом максимальное значение радиуса составляло $8 \mathrm{~mm}$, что в $4-8$ раз ниже критического значения, после которого пламя становится неустойчивым, поэтому фронт пламени можно считать гладким, и его скорость описывается по формуле Маркштейна [34]:

$$
S=S_{L}-L_{M c} K_{c}-L_{M s} K_{s},
$$

где $S_{L}-$ фундаментальная скорость ламинарного горения, $L_{M c}$ - число Маркштейна для учета кривизны 
Таблица 1. Разброс параметров горения газовых смесей

\begin{tabular}{c|c|c|c|c|c|c}
\hline Смесь & $\begin{array}{c}\text { Концентрация } \\
\text { горючего, \% }\end{array}$ & $S_{L}, \mathrm{~m} / \mathrm{s}$ & $\mathrm{Le}$ & $\mathrm{Ze}$ & $\Theta$ & $\begin{array}{c}L_{M}, \\
10^{-5} \mathrm{~mm}\end{array}$ \\
\hline $\mathrm{H}_{2}-$ воздух & $23.30 \pm 0.22$ & $1.36 \pm 0.03$ & $0.394 \pm 0.001$ & $3.17 \pm 0.02$ & $6.14 \pm 0.04$ & $1.63 \pm 0.06$ \\
\hline $\mathrm{C}_{2} \mathrm{H}_{4}-$ воздух & $8.00 \pm 0.09$ & $0.700 \pm 0.007$ & $0.771 \pm 0.001$ & $9.81 \pm 0.03$ & $8.34 \pm 0.01$ & $7.62 \pm 0.05$ \\
\hline $\mathrm{C}_{3} \mathrm{H}_{8}-$ воздух & $5.20 \pm 0.06$ & $0.330 \pm 0.052$ & $0.943 \pm 0.027$ & $9.54 \pm 0.05$ & $7.81 \pm 0.01$ & $4.25 \pm 0.12$
\end{tabular}

Таблица 2. Критические радиусы пламени и числа Маркштейна для рассматриваемых газовых смесей

\begin{tabular}{c|c|c|c}
\hline Смесь & $\begin{array}{c}\text { Концентрация } \\
\text { горючего, \% }\end{array}$ & $M a_{b}$ & $r_{c}, \mathrm{~mm}$ \\
\hline $\mathrm{H}_{2}$-воздух & $23.30 \pm 0.22$ & $3.32 \pm 0.2$ & $31.6 \pm 0.4$ \\
\hline $\mathrm{C}_{2} \mathrm{H}_{4}$-воздух & $8.00 \pm 0.09$ & $14.93 \pm 0.35$ & $60.2 \pm 0.5$ \\
\hline $\mathrm{C}_{3} \mathrm{H}_{8}$-воздух & $5.20 \pm 0.06$ & $5.47 \pm 0.56$ & $67.9 \pm 6.2$
\end{tabular}

пламени, $K_{c}-$ стретч-фактор, связанный с кривизной пламени, $L_{M s}$ - число Маркштейна для учета растяжения пламени расходящимся потоком горючей смеси, $K_{s}$ - стретч-фактор, связанный с растяжением пламени расходящимся потоком.

В общем случае значения и различаются, соответственно стретч-фактор рассчитывается для каждого механизма растяжения пламени отдельно $[35,36]$. В оценочных расчетах часто используют одно значение длины Маркштейна, соответствующее общему стретч-фактору $K=K_{c}+K_{s}$. С учетом теплового расширения продуктов сгорания видимая скорость пламени рассчитывается по формуле

$$
S_{v}=d R_{f} / d t=\Theta\left(S_{L}-L_{M} K\right)=\Theta\left(S_{L}-L_{M} \frac{2}{R_{f}} \frac{d R_{f}}{d t}\right) .
$$

Из формулы (2) можно получить зависимость видимой скорости в явном виде

$$
\frac{d R_{f}}{d t}=\frac{\Theta S_{L}}{1+\Theta L_{M}\left(2 / R_{f}\right)} .
$$

С учетом погрешности состава смеси, а следовательно, и соответствующих характеристик горения, теоретически возможный по формуле (3) разброс скоростей распространения пламени в экспериментах [1] составляет до $2.7 \%$ в смеси $\mathrm{H}_{2}$-воздух, $2 \%$ в смеси $\mathrm{C}_{2} \mathrm{H}_{4}$-воздух и $15 \%$ в смеси $\mathrm{C}_{3} \mathrm{H}_{8}$-воздух. Дифференцируя представленные в работе [1] зависимости радиуса пламени от времени, можно получить разброс скоростей пламени при значениях радиуса $3 \mathrm{~mm}$ до 2\% для смеси $\mathrm{H}_{2}$-воздух, 4\% для смеси $\mathrm{C}_{2} \mathrm{H}_{4}$-воздух и $12 \%$ для смеси $\mathrm{C}_{3} \mathrm{H}_{8}-$ воздух.

Таким образом, разброс скоростей, наблюдаемый при значениях радиуса пламени до $8 \mathrm{~mm}$, может быть объ- яснен погрешностью состава смеси и, как указывают авторы, различием энергии инициирования.

\section{2. Экспериментальная часть}

В ходе наших экспериментов по исследованию ускорения пламени в изначально неподвижных бедных водородно-воздушных смесях постоянного состава (один баллон) было обнаружено, что при идентичных начальных условиях эксперимента скорость фронта пламени в каждый момент времени может отличаться на величину, составляющую до $17 \%$ от среднего значения [5].

В работах [4,5] представлены экспериментальные зависимости среднего радиуса фронта пламени. Однако опубликованные данные были уточнены с использованием усовершенствованной методики обработки теневых изображений. Зависимости среднего радиуса и средней скорости фронта пламени представлены на рис. 1.

Безусловно, существенное влияние оказывает состав исследуемой смеси. Водородно-воздушная смесь в работе [1] содержит 23.3\% водорода, тогда как исследуемая в настоящей работе смесь содержит 15\% водорода. Исходя из графиков работы [1], нетрудно заметить, что при достижении радиуса пламени $3 \mathrm{~mm}$ скорости пламен с разной энергией инициирования начинают стремиться к одинаковому постоянному значению. Это свидетельствует о том, что само по себе гладкое расширяющееся пламя менее подвержено стохастическим процессам неустойчивости. Более того, различается количество экспериментальных точек при радиусе пламени меньше $10 \mathrm{~mm}$ в работах [1] и данной. В то же время наши эксперименты проводились в большем масштабе, аналогичных точек у нас в разы меньше. Важно отметить, что для различных задач начальный этап развития фронта пламени будет иметь различный масштаб. В случае исследования больших объемов, как например, в работе [5], в качестве начального этапа может быть принято пламя радиусом порядка единиц сантиметров.

Авторами было высказано предположение, что полученный разброс имеет фундаментальный характер и объясняется случайным распределением начальных возмущений, из которых развивается картина складчатого неустойчивого пламени. Максимальная погрешность эксперимента, обусловленная ошибками измерения времени и радиуса фронта пламени, достигает $2 \%$, разброс 

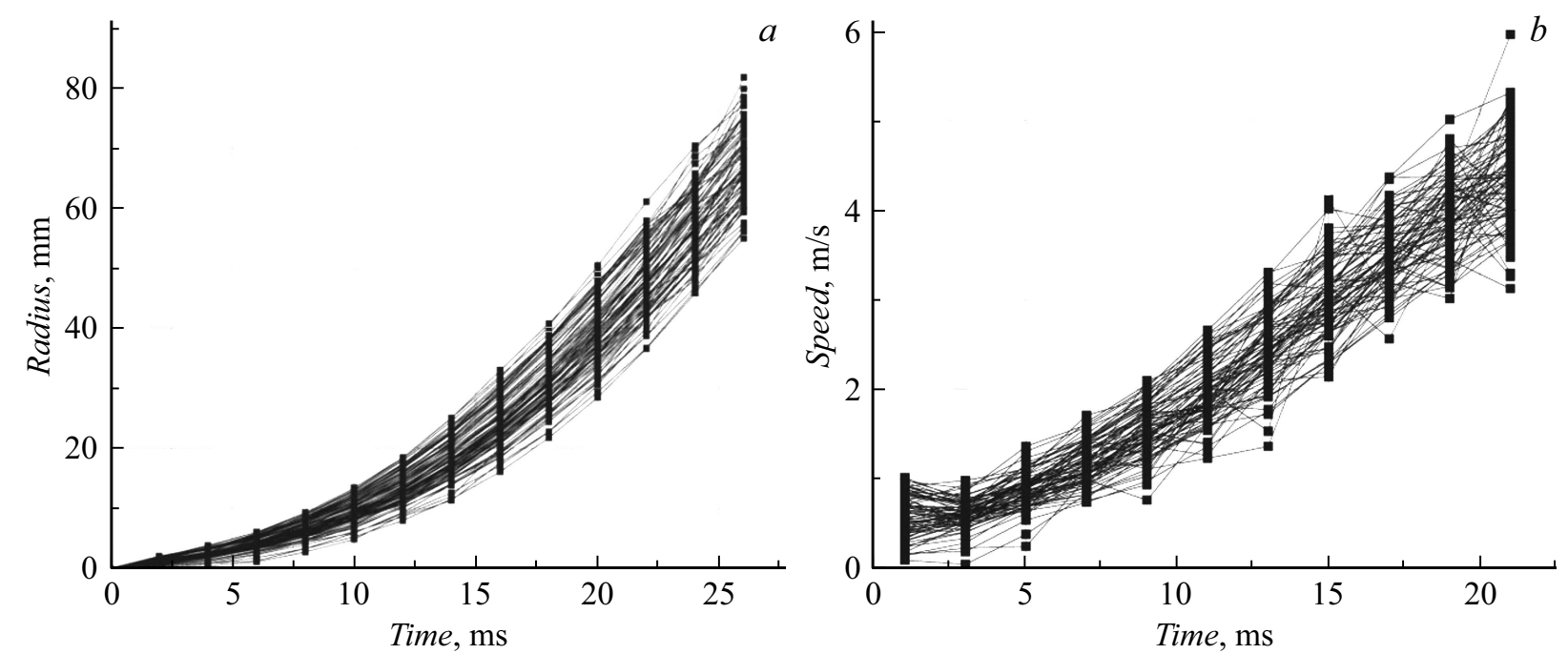

Рис. 1. Зависимости среднего радиуса $(a)$ и средней скорости $(b)$ в 83 экспериментах по распространению сферического фронта водородно-воздушного пламени.

данных достигает $17 \%$, что не может быть объяснено неточностью эксперимента. В настоящей работе также представлены результаты численного моделирования распространения плоского фронта пламени в двумерной постановке, демонстрирующие различие динамики фронта пламени в зависимости от размера и расположения начальных возмущений.

\section{3. Интегрирование уравнения Сивашинского}

Моделирование проводилось путем численного решения уравнения Сивашинского [12], описывающего динамику функции возмущений неустойчивого фронта пламени в газовых смесях. В классической форме уравнение Сивашинского может быть записано в виде

$$
\begin{aligned}
& \frac{\partial \Phi}{\partial t}+Z e^{2}(1-L e)^{2} \nabla^{4} \Phi+\left(\frac{1}{2} Z e(1-L e)-a\right) \nabla^{2} \Phi \\
& +\frac{1}{2}(\nabla \Phi)^{2}=\frac{\Theta-1}{8 \pi^{2} \Theta} \operatorname{Re}\left(\int_{-\infty}^{\infty}|k| \exp (i k(x-\xi)) \Phi(\xi) d k d \xi\right),
\end{aligned}
$$

где $\Phi$ - положение фронта пламени, $Z e-$ число Зельдовича, $L e$ - число Льюиса, $\Theta$ - степень расширения продуктов сгорания.

Пространственные переменные нормированы на тепловую толщину фронта пламени $\delta_{\mathrm{th}}=\chi / S_{L}$, а время на $\chi /\left(S_{L}\right)^{2}$, где $\chi-$ температуропроводность горючей смеси, $S_{L}$ - нормальная скорость плоского пламени.

Поскольку уравнение Сивашинского описывает функцию возмущений фронта пламени, для расчета перемещения пламени аналогично случаю полуограниченного пространства, на каждом расчетном шаге к зависимо- сти $\Phi(x)$ прибавлялось постоянное смещение

$$
\langle\Phi(x)\rangle=S_{L}\left(A / A_{0}(\Theta-1)+1\right) \Delta t .
$$

Поверхность, описываемая уравнением Сивашинского, неустойчива. Различают два механизма неустойчивости пламени в данной постановке задачи: термодиффузионный и гидродинамический (Дарье-Ландау). Малые возмущения растут по термодиффузионному механизму до достижения некоторого критического размера. Дальнейший рост возмушений определяется механизмом Дарье-Ландау [37]. Длину волны возмущения, при которой наблюдается максимальная скорость роста по механизму Дарье-Ландау, можно определить по формуле из работы [28]:

$$
\lambda_{D L}=2 \pi \delta_{\mathrm{th}}\left(1+\frac{(\Theta+1)}{(\Theta-1)^{2}} \Theta \ln \Theta\right) .
$$

В настоящей работе расчеты проводились по методике, описанной в [15], с поправкой на возросшую производительность вычислительной техники. Фронт пламени описывался в координатах $x-z$, где $x$ - координата по начальному гладкому фронту, $z$ - координата, направленная по нормали к $x$. Рассматривался участок изначально прямого фронта пламени длиной $L=1 \mathrm{~m}$ в водородно-воздушной смеси с содержанием водорода $15 \%$. Нормальная скорость плоского пламени в такой смеси $S_{L}=0.34 \mathrm{~m} / \mathrm{s}$, числа Льюиса и Зельдовича $L e=0.36, Z e=4$, степень расширения продуктов сгорания $-\Theta=4.59$. Значение температуропроводности $\chi=3.26 \cdot 10^{-5} \mathrm{~m}^{2} / \mathrm{s}$ получено интерполяцией табличных данных для газовых смесей. Устанавливались периодические граничные условия. В начальный момент времени форма фронта пламени задавалась прямой с возмущениями, либо без них в случае теста на „собственные шумы“ расчетной модели. Шаг по времени и пространству выбирался аналогично [15]. Для проверки сходимости при 


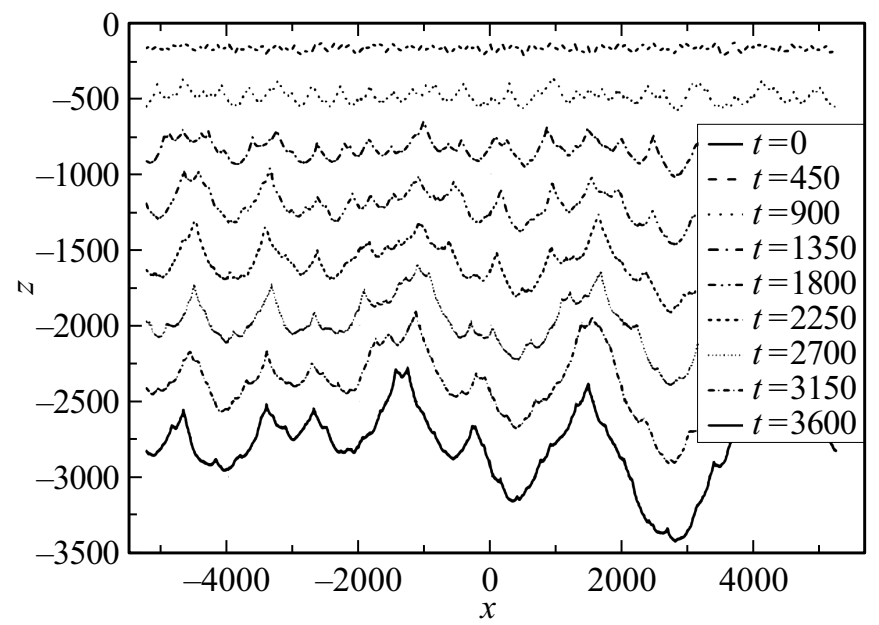

Рис. 2. Эволюция плоского фронта пламени со случайным распределением начальных возмущений в 15\% водородновоздушной смеси. Время и расстояние - в безразмерных единицах.

изменении шага интегрирования был проведен расчет в классической постановке с начальными возмущениями в виде прямых участков длиной $25 \delta_{\text {th }}$ со случайным распределением высоты до $10^{-3} \delta_{\text {th }}$ (рис. 2). Начальное распределение возмущений в данном случае задавалось разбиением расчетной области на участки по 25 ячеек, высота которых задавалась с помощью генератора случайных чисел. В момент времени 3600 безразмерных единиц амплитуда колебаний значений $\Phi$ составляет 1152 безразмерных единиц длины, что соответствует приблизительно $110 \mathrm{~mm}$. Уменьшение шага по времени и по пространству в 2 раза приводит к незначительному изменению решения $(<1 \%)$. При отсутствии начальных возмущений фронт пламени распространяется прямым в течение всего расчетного периода, что свидетельствует об отсутствии влияния „расчетных шумов“ на решение.

Для количественной характеристики влияния искривления фронта пламени на скорость горения используется коэффициент складчатости [13,38]. Детально влияние избытка площади искривленного фронта на скорость горения разобрано в [24].

С использованием разработанного кода была проведена серия расчетов эволюции фронта пламени с различающимися начальными возмущениями. Поскольку расчетный метод лишен собственных „шумов“ в случае плоского фронта пламени, а в природе распространение пламени сопровождается возмущениями, вызванными неоднородностью источника инициирования, остаточными течениями и флуктуациями давления газа, в расчетах были заданы искусственно различные начальные возмущения. В качестве начальных возмущений использовались: прямоугольные возмущения случайной высоты с шириной 0.25 и $1 \lambda_{\mathrm{DL}} \mathrm{c}$ амплитудой до $0.001 \delta_{\mathrm{th}}$, распределенные по всему фронту (кривые 1 и 2 на рис. 3,a); одиночное прямоугольное возмущение с шириной $0.5,1$ и $2 \lambda_{\mathrm{DL}} \mathrm{c}$ амплитудой $0.1 \delta_{\text {th }}$ (кривые $3-5$ на рис. $3, b$ ); один положительный гармонический полупериод с шириной $0.5,1$ и $2 \lambda_{\mathrm{DL}}$ с амплитудой $0.1 \delta_{\text {th }}$ (кривые $6-8$ на рис. $3, b$ ); два прямоугольных возмущения с шириной $0.5,1$ и $2 \lambda_{\mathrm{DL}}$ с амплитудой $0.1 \delta_{\text {th }}$ и промежутком, равным ширине возмущения (кривые 9-11 на рис. $3, b$ ); прямоугольные возмущения случайной высоты с шириной $0.5,1$ и $2 \lambda_{\mathrm{DL}} \mathrm{c}$ амплитудой до $0.1 \delta_{\mathrm{th}}$, распределенные по всему фронту (кривые $12-14$ на рис. $3, b$ ); одиночное прямоугольное возмущение с шириной $\lambda_{\mathrm{DL}}$ и амплитудой $30 \delta_{\mathrm{th}}$ (кри-
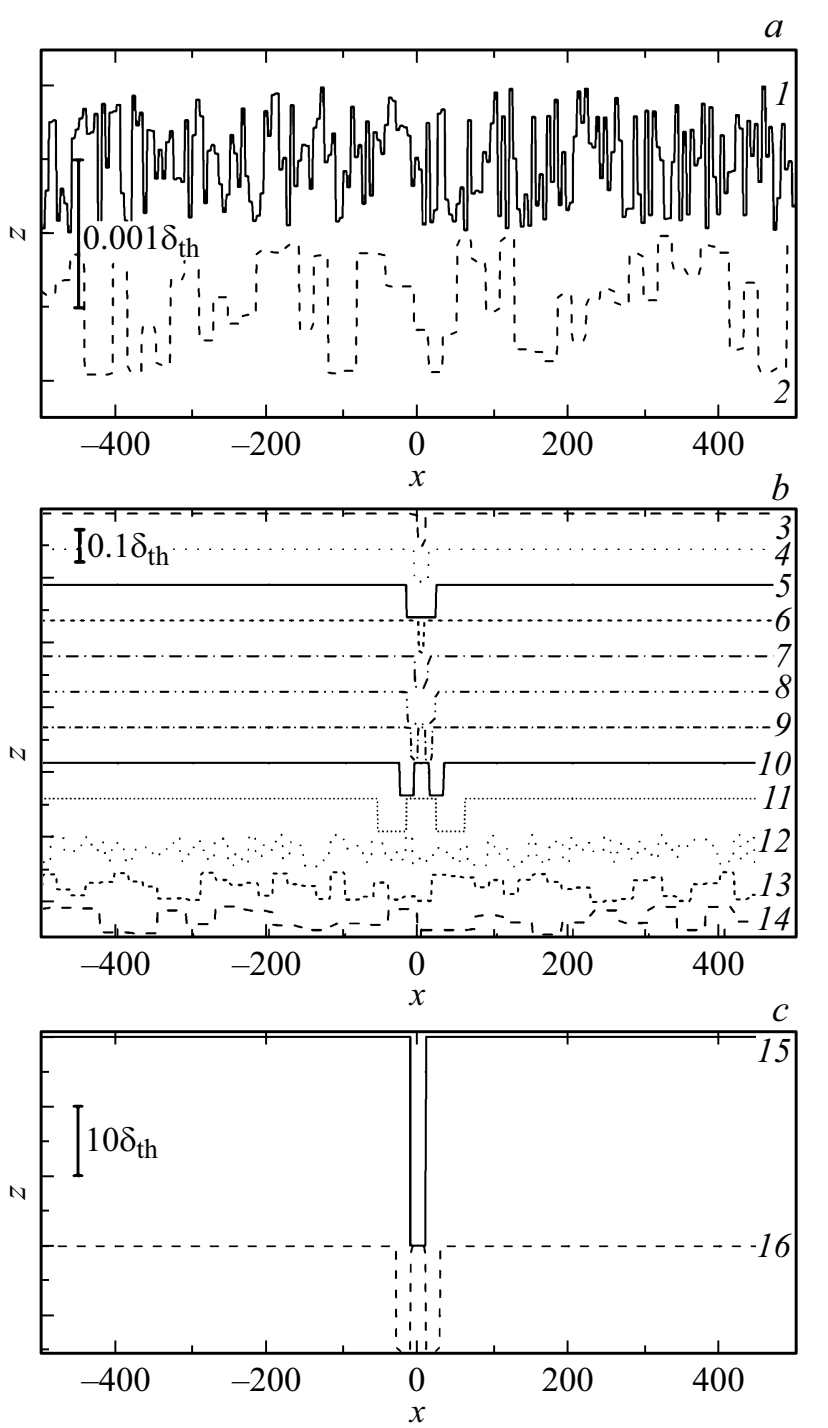

Рис. 3. Формы фронта пламени с начальными возмущениями: $a-1,2-$ возмущения с длиной волны 0.25 и $1 \lambda_{\mathrm{DL}}$, случайной амплитудой до $0.001 \delta_{\mathrm{th}}$, распределенные по всему фронту; $b$ - возмущения с амплитудой до $0.1 \delta_{\mathrm{th}} ; 3-5-$ единичные с длиной волны $0.5,1$ и $2 \lambda_{\mathrm{DL}} ; 6-8-$ аналогично $3-5$, гармонические полупериоды; 9-11 - аналогично 3-5, сдвоенные прямоугольные возмущения; $12-14-$ возмущения с длиной волны $0.5,1$ и $2 \lambda_{\mathrm{DL}}$, распределенные по всему фронту; $c$ 15,16 - одиночное и сдвоенное возмущения с длиной волны $\lambda_{\mathrm{DL}}$ и амплитудой 30 и $15 \delta_{\mathrm{th}}$. 

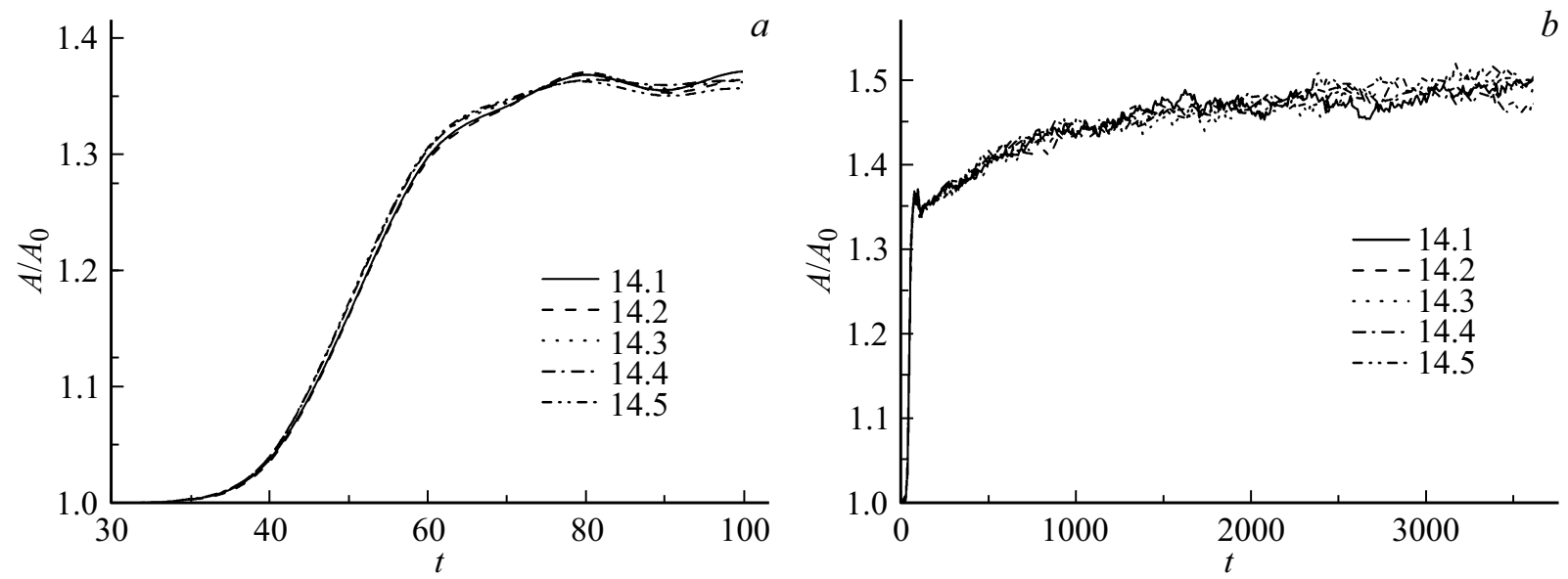

Рис. 4. Зависимости значений коэффициента складчатости от времени с отличающимися полями начальных возмущений в виде прямоугольных возмущений случайной высоты с шириной $2 \lambda_{\mathrm{DL}} \mathrm{c}$ амплитудой до $0.1 \delta_{\mathrm{th}}$, распределенных по всему фронту (соответствует 14 на рис. 3) в двух временных масштабах, соответствующих периоду быстрого роста коэффициента складчатости $(a)$ и полному времени расчета $(b)$.

вая 15 на рис. 3,c); два прямоугольных возмущения c шириной $\lambda_{\mathrm{DL}}$ и амплитудой $15 \delta_{\mathrm{th}}$ и промежутком, равным ширине возмущения (кривая 16 на рис. $3, c$ ).

Для каждой начальной конфигурации фронта пламени были рассчитаны его формы в последовательные моменты времени. Из полученных функций, описывающих фронт пламени в каждый момент времени, были вычислены значения коэффициента складчатости как отношения длины искривленного фронта пламени $A$ к длине гладкого фронта $A_{0}=L$. Длина искривленного фронта пламени рассчитывалась по формуле

$$
A=\int_{0}^{L} \sqrt{1+\left(\frac{\partial \Phi}{\partial x}\right)^{2}} d x .
$$

Для начальных конфигураций фронта, соответствующих видам 1,2,12,13 и 14 на рис. 3, было проведено по 5 расчетов с отличающимися полями начальных возмущений при заданных значениях длины волны и максимальной амплитуды. Все расчеты показали близкие зависимости коэффициента складчатости от времени для каждого вида начальных возмущений. На рис. 4 приведена зависимость для начальных возмущений в виде прямоугольных возмущений случайной высоты с шириной $2 \lambda_{\mathrm{DL}} \mathrm{c}$ амплитудой до $0.1 \delta_{\mathrm{th}}$, распределенных по всему фронту (соответствует 14 на рис. 3) в двух временных масштабах, соответствующих периоду быстрого роста коэффициента складчатости (рис. 4, $a$ ) и полному времени расчета (рис. $4, b)$.

Зависимости, представленные на рис. 4, показывают, что для однотипных случайных начальных возмущений моменты времени начала и окончания резкого роста коэффициента складчатости практически совпадают (рис. $4, a)$. В момент окончания резкого роста значение коэффициента складчатости близко к 1.35. Дальнейшее увеличение происходит схожим образом (рис. $4, b)$.

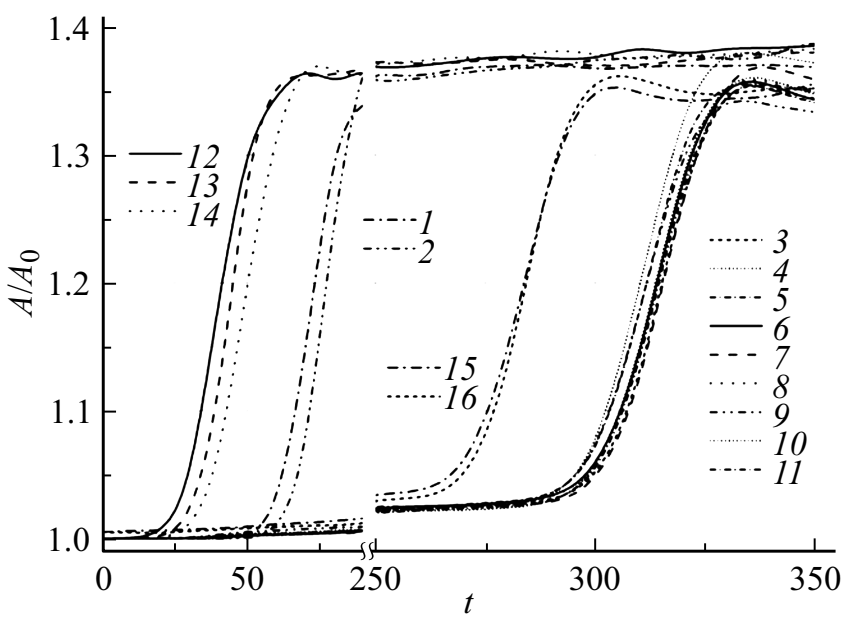

Рис. 5. Зависимости значений коэффициента складчатости от времени. Обозначения кривых аналогичны приведенным на рис. 3. Время - в безразмерных единицах.

Сравнение зависимостей значений коэффициента складчатости от времени для всех видов начальных условий, показанных на рис. 3 , представлено на рис. 5 .

Полученные зависимости показывают, что момент начала развития складчатой поверхности пламени различается в зависимости от начальных возмущений фронта.

Для всех начальных условий после определенного периода индукции наблюдается резкий рост коэффициента складчатости до значения около 1.35. Далее скорость роста зависит от начальных условий незначительно. Кратчайшее время индукции, когда начальные возмущения случайной амплитуды до $0.1 \delta_{\text {th }}$ распределены по всей поверхности пламени (кривые 12-14 на рис. 5). Уменьшение амплитуды начальных возмущений в 100 раз увеличивает время индукции примерно в 1.5 раза (кривые 1,2 на рис. 5). Время индукции фронта пламени 
с одиночными и двойными возмущениями амплитуды $0.1 \delta_{\text {th }}$ примерно в 15 раз больше (кривые 3-11 на рис. 5). Увеличение амплитуды одиночного возмущения в 300 раз либо двойного в 150 раз сокращает время индукции незначительно (кривые 15, 16 на рис. 5). Ширина и форма начального возмущения при постоянной амплитуде практически не влияют на время индукции и скорость роста коэффициента складчатости. При дальнейшем увеличении коэффициента складчатости пламени наблюдается незначительный разброс зависимостей.

Как было показано в работе [38], скорость неустойчивого расширяющегося пламени может быть оценена как:

$$
v_{f}=S_{L} A / A_{0}(\Theta-1)+S_{L} .
$$

В момент времени $25 \mathrm{~ms}$, что соответствует 90 безразмерным единицам времени в расчете, величина коэффициента складчатости меняется от 1.006 до 1.345 , что дает в результате разброс скоростей распространения пламени $17.7 \%$ от среднего значения при различных начальных возмущениях фронта пламени. Различие в значениях скорости обусловлено разницей во времени начала резкого роста коэффициента складчатости в зависимости от конфигурации начальных возмущений. Аналогичная динамика ускорения сферически расширяющегося пламени приводит к различным значениям радиуса, при которых начинается интенсивное ускорение фронта пламени, и соответственно определяет весь дальнейший ход развития горения. Полученный результат хорошо согласуется с экспериментальными данными.

\section{Выводы}

Из представленных результатов эксперимента, численного моделирования и их анализа можно сделать следующие выводы:

- распространение искривленного фронта пламени в изначально неподвижной газовой смеси возможно с различными скоростями при неизменных начальных условиях;

- при размерах фронта пламени, соответствующих устойчивому распространению гладкого фронта, этот факт объясняется различиями в энергии и форме поджигающего искрового разряда;

- при бо́льших размерах фронта пламени, соответствующих неустойчивому распространению складчатого фронта, различия вызваны как погрешностью составлении смеси, так и разными конфигурациями начальных возмущений, определяющими динамику развития структуры пламени, и соответственно его скорости;

Приведенные результаты доказывают необходимость статистического описания ускорения ламинарного пламени и развития газовых взрывов.

\section{Конфликт интересов}

Авторы заявляют, что у них нет конфликта интересов.

\section{Список литературы}

[1] S. Essmann, D. Markus, H. Grosshans, U. Maas. Combust. Flame, 211, 44 (2020).

DOI: 10.1016/j.combustflame.2019.09.021

[2] М.Ф. Иванов, А.Д. Киверин, А.Е. Смыгалина, В.М. Зайченко. ЖТФ, 88 (1), 147 (2018). DOI: $10.21883 /$ JTF.2018.01.45499.2271

[3] А.М. Старик, П.С. Кулешов, Н. Титова. ЖТФ, 78 (2), 95 (2008).

[4] В.В. Володин, В.В. Голуб, А.Е. Ельянов, А.Е. Коробов, А.Ю. Микушкин. Вестник ОИВТ РАН, 1 (1), 78 (2018). DOI: $10.33849 / 2018117$

[5] В.В. Володин, В.В. Голуб, А.Е. Ельянов, А.Е. Коробов, А.Ю. Микушкин, В.А. Петухов. Вестник МГТУ им. Н.Э. Баумана. Сер. Естественные науки, 83 (2), 64 (2019). DOI: 10.18698/1812-3368-2019-2-64-80

[6] C. Xu, A.A. Konnov. Energy, 43, 19 (2012). DOI: $10.1016 /$ j.energy.2011.11.006

[7] F.N. Egolfopoulos, N. Hansen, Y. Ju, K. Kohse-Höinghaus, C.K. Law, F. Qi. Prog. Energ. Combust., 43, 36 (2014). DOI: 10.1016/j.pecs.2014.04.004

[8] Ю.В. Полежаев, И.Л. Мостинский. ТВТ, 43 (6), 933 (2005). DOI: $10.1007 / \mathrm{s} 10740-005-0141-1$

[9] G.O. Thomas, G.L. Oakley. Chem. Eng.-Lond., 71, 187 (1993).

[10] J.E. Shepherd, J.H.S. Lee. Major Research Topics in Combustion (Springer-Verlag, Hampton, VA, 1992) DOI: 10.1007/978-1-4612-2884-4_22

[11] S.P.M. Bane, J.L. Ziegler, P.A. Boettcher, S.A. Coronel, J.E. Shepherd, J. Loss. Prevent. Proc., 26, 290 (2013). DOI: $10.1016 /$ j.jlp.2011.03.007

[12] G.I. Sivashinsky. Acta Astronaut., 4, 1177 (1977). DOI: 10.1016/0094-5765(77)90096-0

[13] L. Filyand, G. Sivashinsky, M. Frankel. Physica D, 72, 110 (1994). DOI: 10.1016/0167-2789(94)90170-8

[14] M. Frankel, G. Sivashinsky. J. Phys., 48, 25 (1987). DOI: $10.1051 /$ jphys:0198700480102500

[15] D.M. Michelson, G.I. Sivashinsky. Acta Astronaut., 4, 1207 (1977). DOI: 10.1016/0094-5765(77)90097-2

[16] D. Michelson, G. Sivashinsky. Combust. Flame, 48, 211 (1982). DOI: 10.1016/0010-2180(82)90128-6

[17] O. Kupervasser, Z. Olami, I. Procaccia. Phys. Rev. Lett., 76 (1), 146 (1996). DOI: 10.1103/PhysRevLett.76.146

[18] С.С. Минаев, Е.А. Пирогов, О.В. Шарыпов. ФГВ, 5, 8 (1996). DOI: 10.1007/BF01998569

[19] I. Brailovsky, P.V. Gordon, L. Kagan, G. Sivashinsky. Combust. Flame, 162, 2077 (2015). DOI: 10.1016/j.combustflame.2015.01.006

[20] J. Yanez, M. Kuznetsov. Phys. Lett. A, 380, 2549 (2016). DOI: 10.1016/j.physleta.2016.05.048

[21] A. Wähner, G. Gramse, T. Langer, M. Beyer, J. Loss. Prevent. Proc., 26 (6), 1655 (2013). DOI: 10.1016/j.jlp.2013.06.002

[22] Bronkhorst High-Tech B.V., EL-FLOW Select Digital Thermal Mass Flow Meters and Controllers for Gases (Ruurlo Netherlands: Bronkhorst, 2019)

[23] Servomex Group Limited, Servomex Analyzers. Product overview: MiniHD (5200). (Crowborough, United Kingdom: Servomex Group Ltd, 2018).

[24] C.J. Sun, C.J. Sung, L. He, C.K. Law, Combust. Flame, 118 (1-2), 108 (1999). DOI: 0.1016/S0010-2180(98)00137-0

[25] O.G. Penyazkov, K.L. Sevrouk, V. Tangirala, N. Joshi. P. Combust. Inst. 32, 2421 (2009). DOI: $10.1016 /$ j.proci.2008.06.194 
[26] G.P. Smith, D.M. Golden, M. Frenklach, N.W. Moriarty, B. Eiteneer, M. Goldenberg, C.T. Bowman, R.K. Hanson, S. Song, J.W.C. Gardiner, V.V. Lissianski, Z. Qin. GRI-Mech 3.0. (Online. Available: http://combustion.berkeley.edu/gri$\mathrm{mech} /$ version30/text30.html2019).

[27] G. Jomaas, X.L. Zheng, D.L. Zhu, C.K. Law. P. Combust. Inst., 30, 193 (2005). DOI: 10.1016/j.proci.2004.08.228

[28] G. Joulin, T. Mitani. Combust. Flame, 40, 235 (1981). DOI: $10.1016 / 0010-2180(81) 90127-9$

[29] K.T. Aung, M.I. Hassan, G.M. Faeth. Combust. Flame, 109 (1-2), 1 (1997). DOI: 10.1016/S0010-2180(96)00151-4

[30] L.-K. Tseng, M.A. Ismail, G.M. Faeth. Combust. Flame, 95 (4), 410 (1993). DOI: 10.1016/0010-2180(93)90007-P

[31] S.G. Davis, J. Quinard, G. Searby. Combust. Flame, 130 (1-2), 123 (2002). DOI: 10.1016/S0010-2180(02)00368-1

[32] J. K. Bechtold, M. Matalon. Combust. Flame, 67 (1), 77 (1987). DOI: 10.1016/0010-2180(87)90015-0

[33] W. Kim, Y. Sato, T. Johzaki, T. Endo. J. Loss Prevent. Proc., 60, 264 (2019). DOI: 10.1016/j.jlp.2019.05.008

[34] G.H. Markstein. J. Aeronaut. Sci., 18 (3), 199 (1951). DOI: $10.2514 / 8.1900$

[35] D. Bradley, P.H. Gaskell, X.J. Gu. Combust. Flame, 104, 176 (1996). DOI: 10.1016/0010-2180(95)00115-8

[36] D. Bradley, R.A. Hicks, M. Lawes, C.G.W. Sheppard, R. Woolley. Combust. Flame, 115 (1-2), 126 (1998). DOI: $10.1016 / 0010-2180(95) 00115-8$

[37] Л.Д. Ландау. ЖЭТФ, 14, 240 (1944). DOI: 10.1016/B978-0-08-092523-3.50044-7

[38] V. Golub, A. Elyanov, A. Korobov, A. Mikushkin, V. Petukhov, V. Volodin. ETFS, 109, 109845 (2019).

DOI: 10.1016/j.expthermflusci.2019.109845 